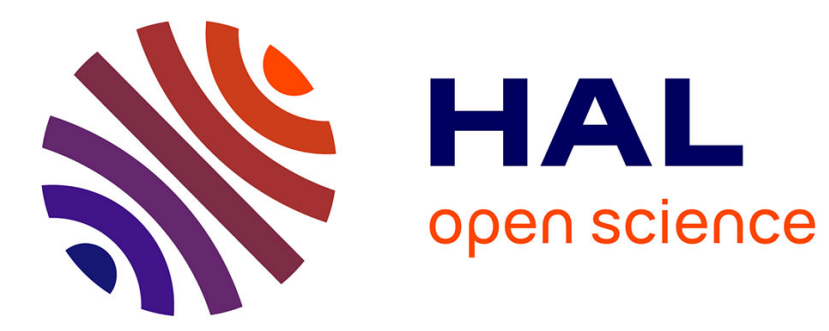

\title{
The deubiquitinating enzyme DUB2A enhances CSF3 signalling by attenuating lysosomal routing of CSF3 receptor
}

Annemarie Meenhuis, Carola Verwijmeren, Onno Roovers, Ivo Paul Touw

\section{- To cite this version:}

Annemarie Meenhuis, Carola Verwijmeren, Onno Roovers, Ivo Paul Touw. The deubiquitinating enzyme DUB2A enhances CSF3 signalling by attenuating lysosomal routing of CSF3 receptor. Biochemical Journal, 2011, 434 (2), pp.343-351. 10.1042/BJ20101628 . hal-00565907

\section{HAL Id: hal-00565907 https://hal.science/hal-00565907}

Submitted on 15 Feb 2011

HAL is a multi-disciplinary open access archive for the deposit and dissemination of scientific research documents, whether they are published or not. The documents may come from teaching and research institutions in France or abroad, or from public or private research centers.
L'archive ouverte pluridisciplinaire HAL, est destinée au dépôt et à la diffusion de documents scientifiques de niveau recherche, publiés ou non, émanant des établissements d'enseignement et de recherche français ou étrangers, des laboratoires publics ou privés. 


\title{
THE DEUBIQUITINATING ENZYME DUB2A ENHANCES CSF3 SIGNALLING BY ATTENUATING LYSOSOMAL ROUTING OF CSF3 RECEPTOR
}

Annemarie Meenhuis, Carola Verwijmeren, Onno Roovers and Ivo P. Touw, Department of Hematology, Erasmus University Medical Center, Rotterdam, The Netherlands

Address correspondence to: Ivo P. Touw, PhD. Department of Hematology, Erasmus University Medical Center, Dr Molewaterplein 50, 3015 GE, Rotterdam, The Netherlands; Phone: +31-10-7043837; Fax: +31-10-7044745; E-mail: i.touw@erasmusmc.nl

Key words: endocytosis, granulocyte colony-stimulating factor (CSF3) receptor, deubiquitinating enzyme (DUB), signal transducer and activator of transcription (STAT) 5, suppressor of cytokine signalling (SOCS) 3

Running title: DUB2A controlled CSF3 receptor signalling

\begin{abstract}
Abbreviations: AMSH, associated molecule with the SH3 domain of STAM; Bio-CSF3, biotinylated granulocyte colony-stimulating factor; CSF3, granulocyte colony-stimulating factor; CSF3R, CSF3 receptor; DOR, $\delta$-opioid peptide receptor; DUB, deubiquitinating enzyme; EGFR, epidermal growth-factor receptor; ER, endoplasmic reticulum; ESCRT, endosomal sorting required for transport; IL, interleukin; JAK, janus tyrosine kinase; JAMM, JAB1/MPN/mov34-domain-containing; MAPK, mitogen-activated protein kinase; MIT, microtubule interacting and transport; $\mathrm{PAR}_{2}$, protease activated receptor 2; $\mathrm{SH} 2$, src homology 2; SOCS3, suppressor of cytokine signalling 3; STAM, signal transducing adaptor molecule; STAMBP, STAM binding protein; STAT, signal transducer and activator of transcription; Ub, ubiquitin; UBPY, ubiquitin isopeptidase Y; USP, ubiquitin specific peptidase; wt, wild-type.
\end{abstract}




\section{SYNOPSIS}

Ubiquitination of the colony-stimulating factor 3 receptor (CSF3R) occurs after activated CSF3Rs are internalized and reside in early endosomes. CSF3R ubiquitination is crucial for lysosomal routing and degradation. The E3 ligase suppressor of cytokine signalling 3 (SOCS3) has been shown to play a major role in this process. Deubiquitinating enzymes remove ubiquitin moieties from target proteins by proteolytic cleavage. Two of these enzymes, AMSH and UBPY, interact with the general endosomal sorting machinery. Whether deubiquitinating enzymes control CSF3R trafficking from early towards late endosomes is unknown. In the present study, we asked whether AMSH, UBPY or a murine family of deubiquitinating enzymes could fulfil such a role. This DUB family comprises four members (DUB1, DUB1A, DUB2, and DUB2A), which were originally described as being hematopoietic-specific and cytokine inducible, but their function in cytokine receptor routing and signalling has remained largely unknown. Here, we show that DUB2A expression is induced by CSF3 in myeloid 32D cells and that DUB2 decreases ubiquitination and lysosomal degradation of the CSF3R, leading to prolonged signalling. These data support a model in which CSF3R receptor ubiquitination is dynamically controlled at the early endosome by feedback mechanisms involving CSF3-induced E3 ligase (SOCS3) and deubiquitinase (DUB2A) activities. 


\section{INTRODUCTION}

To control the duration of signalling, many activated growth-factor receptors are subject to ligand-induced endocytosis and intracellular routing to early endosomes and lysosomes, where the receptor proteins are being degraded [1]. Ubiquitination is the major protein modification involved in this process [1-2]. Colony stimulating factor 3 (CSF3) stimulates myeloid cell proliferation and differentiation [3]. CSF3 activates the latent transcription factor signal transducer and activator of transcription 3 (STAT3), which induces the expression of SOCS3 encoding the suppressor of cytokine signalling 3 (SOCS3) protein [4-5]. SOCS3 is recruited to a phosphorylated tyrosine (Y729) motif in the activated CSF3 receptor (CSF3R) [4] and forms an E3 ligase complex via its SOCS box [6]. It was recently shown that SOCS3, by ubiquitination of a juxtamembrane lysine in the CSF3R cytoplasmic domain, promotes CSF3R routing from early to late endosomes, leading to signal termination [7].

Deubiquitinating enzymes remove ubiquitin $(\mathrm{Ub})$ from their target substrates and are therefore potentially important in controlling receptor routing and signalling. Two endosomal deubiquitinating enzymes associated with routing of activated receptors are signal transducing adaptor molecule (STAM) binding protein (STAMBP), also known as associated molecule with the SH3 domain of STAM (AMSH), a member of the JAB1/MPN/mov34-domaincontaining (JAMM) family and ubiquitin specific peptidase 8 (USP8) which is also known as ubiquitin isopeptidase Y (UBPY) a member of the ubiquitin-specific proteases (USP) family of deubiquitinating enzymes [8-9]. Both bind to the endosomal sorting complex required for transport (ESCRT)-0 subunit STAM1 via their SH3 domain and to ESCRT-III through their microtubule interacting and transport (MIT) domains [10-13]. AMSH is involved in endosomal trafficking of tyrosine kinase receptors such as the epidermal growth-factor receptor (EGFR) and G-protein coupled receptors like protease activated receptor $2\left(\mathrm{PAR}_{2}\right)$, $\delta$-opioid peptide receptor (DOR) and CXCR4 [8, 14-17]. UBPY has been shown to be important in stabilizing the ESCRT-0 complex [18-20] and is essential for deubiquitination and degradation of EGFR, PAR 2 and DOR $[14-15,21]$. UBPY has been proposed to maintain the pool of free ubiquitin Ub by releasing it from the cargo just prior to lysosomal degradation [22].

The murine DUB family comprises 4 highly homologous USP proteins (DUB1, DUB1A, DUB2 and DUB2A) that were initially identified in hematopoietic cells [23-25]. Their sequence similarity and location on chromosome 7 suggest that they have arisen from a tandem duplication event [24-25]. All four DUBs are rapidly and transiently induced in response to various cytokines [23-26]. For instance, DUB1 expression is induced by interleukin (IL)3, IL5 and CSF2, cytokines that activate receptors with a $\beta$ common subunit, resulting in a G1 phase cell cycle arrest $[25,27]$. For IL3-induced upregulation of DUB1, a critical role for JAK2 and the Ras/Raf/MAPK pathway was shown [28]. However, these pathways appeared necessary but not sufficient, because JAK2 and MAPK activation by the erythropoietin receptor (EPOR) did not result in increased DUB1 expression [28-29]. DUB1 A is IL3 inducible in B-lymphocytes, whereas DUB2 is induced by IL2 in T-cells [26]. DUB2 inhibits apoptosis after IL2 withdrawal and prolongs STAT5 phosphorylation in Ba/F3 cells expressing IL $2 \mathrm{R} \beta[24,30]$. The $D U B 2 A$ enhancer element contains one Ets and two AP-1 binding sites that are required for $D U B 2 A$ expression in T-lymphocytes [31]. Finally, DUB1 controls ubiquitination of the dynein heavy chain, which is part of the complex that regulates the movement of cargo along microtubules [32]. Together, these findings suggest that DUBs may modulate a variety of processes at distinct intracellular locations, including endosomal cargo trafficking and processing.

The first human family member found to be homologous to the murine DUB family by data base analysis was DUB3. DUB3 was subsequently shown to be IL4 and IL6 inducible and constitutive expression can block cell proliferation [33]. The official name of DUB3 is now USP17L2. The human USP17 family is reported to be encoded within RS447, a megasatellite repeat, on human chromosome 4 and on chromosome 8 embedded within the copy number variable beta-defensin cluster [34-35]. The multiple family members in different 
species have evolved as the result of duplication of common ancestral sequence, which is in general replicated after the species diverged [35-36].

Previously, we have shown that CSF3R ubiquitination, predominantly taking place when the receptor resides in early endosomes, triggers its routing towards late endosomes, followed by lysosomal degradation [7]. In view of this negative feedback mechanism involving CSF3induced SOCS3 expression followed by lysosomal degradation, we asked whether deubiquitinating enzymes acting on CSF3R at the early endosome may antagonize this inhibitory feedback loop. Here, we show that CSF3 induces $D U B 2 A$ expression in myeloid progenitor cells. Subsequently, DUB2A reduces CSF3R ubiquitination and causes the accumulation of CSF3R in early endosomes, leading to prolonged signalling. These results support a model in which DUB2A acts in a positive feedback loop to counterbalance STAT3SOCS3-driven lysosomal targeting of the CSF3R.

\title{
EXPERIMENTAL
}

\section{Cell culture}

293T, Phoenix E and HeLa cells were maintained in Dulbecco's modified Eagle's medium (Gibco DMEM, Invitrogen, Breda, The Netherlands) supplemented with 10\% FCS, $100 \mathrm{IU} / \mathrm{ml}$ penicillin and $100 \mu \mathrm{g} / \mathrm{ml}$ streptomycin at $37^{\circ} \mathrm{C}$ and $5 \% \mathrm{CO}$. HeLa cells were plated on glass cover slips and transiently transfected using lipofectamine (Invitrogen, Breda, The Netherlands). Transfection of $293 \mathrm{~T}$ and Phoenix E cells was done using TransIT-LT1 transfection reagent (Mirus, Madison, WI, USA).

Murine myeloid 32D.cl8.6 cells [37] and Ba/F3//tTA DUB2 [30] were cultured in Roswell Park Memorial Institute Medium (RPMI) 1640 (Gibco, Invitrogen, Breda, The Netherlands) supplemented with $10 \% \mathrm{FCS}, 100 \mathrm{IU} / \mathrm{ml}$ penicillin, $100 \mu \mathrm{g} / \mathrm{ml}$ streptomycin and $10 \mathrm{ng} / \mathrm{ml}$ murine IL3 at $37^{\circ} \mathrm{C}$ and $5 \% \mathrm{CO} 2 . \mathrm{Ba} / \mathrm{F} 3 \beta / \mathrm{tTA}$ DUB2 cells were cultured in presence of 1 $\mu \mathrm{g} / \mathrm{ml}$ doxycycline (Dox, Clontech Laboratories, Mountain View, CA, USA). Dox was replenished every $48 \mathrm{hrs}$. 32D cells and Ba/F3 $\beta /$ tTA DUB2 cells expressing wt CSF3R were obtained by retroviral transduction as previously described [38]. Ba/F3ß/tTA DUB2 cells expressing wt CSF3R were selected by sorting for CSF3R positive cells using a FACSAria flow cytometer (Becton Dickinson, San Diego, CA, USA).

\begin{abstract}
Antibodies
Mouse anti-human CSF3R and mouse anti-human CSF3R coupled to phycoerythrin (PE) were purchased from Becton-Dickinson/PharMingen (San Diego, CA, USA); Rabbit antiFLAG and goat anti-FLAG from Abcam, (Cambridge, UK); Mouse anti-GFP from Roche Applied Sciences (Indianapolis, IN, USA); Goat anti-ACTIN and goat anti-EEA-1 from Santa Cruz Biotechnology inc. (Santa Cruz, CA, USA). Rabbit anti-DUB2 was a gift from James Johnston (University of Belfast) [30]. Secondary goat anti-mouse and goat anti-rabbit either coupled to IRDye680 or IRDye800CW were obtained from LI-COR Biosciences (Lincoln, NE, USA); Donkey anti-goat IRDye 800 from Rockland Immunochemicals (Gilbertsville, PA, USA); Donkey anti-goat 488 (DaG-488) and rabbit anti-goat 546 (RaG-546) from Molecular Probes (Invitrogen, Breda, The Netherlands); Donkey anti-mouse Cy5 (DaM-cy5) and donkey anti-rabbit cy3 (DaR-cy3) from Jackson ImmunoResearch Laboratories, Inc. (West Grove, PA, USA).
\end{abstract}

\section{Expression constructs}


pBABE expression constructs of wt, mKA and K5R CSF3R, tyrosine substitution mutants and the pCDNA3 construct with Flag tagged ubiquitin (Flag-Ub) have all been described previously [7, 39-40]. pME18S-FLAG-DUB2 was a gift from James Johnston (University of Belfast) [30]. pEGFP-C1-AMSH and pEGFP-C1-UBPY were obtained from Sylvie Urbé (University of Liverpool) and have been previously described $[8,18]$.

\section{RNA isolation, DNAse treatment and Quantitative Real Time PCR}

Cells were washed 3 times with Hanks' balanced salt solution (HBSS) and deprived of serum and factors in RPMI at a concentration of 1.5 million cells $/ \mathrm{ml}$ for $4 \mathrm{hrs}$, followed by stimulation with $100 \mathrm{ng} / \mathrm{ml} \mathrm{CSF3}$ for the indicated time points. For the inhibitor study, 10 $\mu \mathrm{M}$ LY294002, $10 \mu \mathrm{M}$ JAK inhibitor I, $10 \mu \mathrm{M}$ SB203580, $10 \mu \mathrm{M}$ U0126, $0.5 \mu \mathrm{M}$ Akt inhibitor IV, $10 \mu \mathrm{M}$ JNK VIII or $10 \mu \mathrm{M}$ PP2 (Calbiochem, Gibbstown, NJ, USA ; all dissolved in DMSO), or DMSO as solvent control was added to the medium $30 \mathrm{~min}$ prior to the CSF3 stimulation. Cells were harvested, resuspended in TRIzol ${ }^{\circledR}$ (Invitrogen, Breda, The Netherlands) and RNA was isolated according to manufacturer's instructions. Removal of genomic DNA, synthesis of cDNA followed by qPCR using Taqman technology (7900HT, PE Applied Biosystems, Foster City, CA, USA) was performed as described before [41]. Primers used for amplification were: mDUB fw222-5'TCTAGCTGACTACATGCTGTC and mDUB rv385-5'GCTTGTGGAAGGCAGAGGTCA, mAMSH-fw1-exon7 5'CCACACGGAGAATGAAGAAG and mAMSH-rv1-exon8 5'TGCGATGGACTCTGGTAAC mUBPY-fw-2545 5'AAGGTGAAGTGGCAGAAGAA and mUBPY-rv-2713 5'CCATCCATGAGGAACAGAAG.

For $\operatorname{Socs} 3$ and the ribonuclease inhibitor (RI), previously published primers were used [41]. To quantify the relative expression of Dub, Amsh, Ubpy and Socs3, the Ct values were normalized for endogenous reference (CtSample $=\mathrm{CtGene}$ of interest $-\mathrm{CtRNase}$ inhibitor) and compared with a calibrator using the $\Delta \Delta \mathrm{Ct}$ method ( $\Delta \Delta \mathrm{Ctsample}=\mathrm{CtSample}-\mathrm{CtCalibrator})$. Expression levels in 32D cells deprived of growth factors for $4 \mathrm{hrs}$ were used as calibrators.

\section{Bio-CSF3 pull downs and CSF3 induced STAT5 phosphorylation}

CSF3R pull down with biotinylated CSF3 (Bio-CSF3) and subsequent SDS-polyacrylamide gel electrophoresis and Western blotting was performed as described [42-43]. Detection of proteins was done using fluorescently labeled secondary antibodies and the Odyssey Infrared Imaging System (LI-COR Biosciences, Lincoln, NE, USA). Quantification was done using the Odyssey Infrared Imaging System software according to the manufacturer's instructions. For off-rates, $\mathrm{Ba} / \mathrm{F} 3 \beta / \mathrm{tTA}$ DUB2 cells stably expressing wt CSF3R were grown in the presence or absence of Dox for 60 hrs. Subsequently cells were cytokine and serum deprived followed by stimulation with CSF3 for $10 \mathrm{~min}$ CSF3 was then washed away and cells were incubated at $37^{\circ} \mathrm{C}$ in RPMI for indicated time points.

\section{Confocal microscopy}

Lysosomal routing of activated membrane CSF3Rs was visualized by confocal laser scanning microscopy (CLSM) using a Leica SP5 instrument as described [7]. In brief, HeLa cells stably expressing wt CSF3R constructs were transfected with different DUB constructs. After $48 \mathrm{hrs}$, cells were incubated with CSF3R antibody and CSF3 for 10 min at RT, washed twice and incubated at $37^{\circ} \mathrm{C}$ for $2 \mathrm{hrs}$. Subsequently, cells were permeabilized with saponin, fixed with 3\% paraformaldehyde and stained for internalized receptors (DaM-cy5), early endosome 
marker EEA1 (DaG-488 or RaG-546) in combination with or without staining for DUB2 (DaR-cy3).

\section{RESULTS}

\section{CSF3 induces Dub2A expression}

To determine if expression of DUB family members is induced by CSF3, we used myeloid 32D cells expressing human CSF3R. Cells were deprived of serum and IL 3 for $4 \mathrm{hrs,}$ followed by CSF3 stimulation for the indicated time points. Dub mRNA expression increased, with maximal levels at $60 \mathrm{~min}$ of CSF3 stimulation (Figure 1A). Nucleotide sequencing identified $D u b 2 A$ as the single member being induced by CSF3. Because DUB2A is highly homologous to DUB2 (97\% identical amino acid sequence), DUB2A was readily detected using a DUB2 antibody, 1 to $3 \mathrm{hrs}$ after initiation of CSF3 stimulation (Figure1C). In contrast, Ubpy and Amsh expression was not induced by CSF3 (Figure 1A). Socs 3 transcript levels, which were assessed in parallel, were maximal at $30 \mathrm{~min}$ of CSF3 stimulation (Figure 1B).

\section{Dub2A induction requires JAK activity}

Previous studies have shown that activation of JAK2 and the Ras/Raf/MAPK pathway is important for IL3 dependent Dub1 induction [28]. To assess which signalling pathways are vital for CSF3-induced $D u b 2 A$ expression, we first focused on the signalling mechanisms coupled to the CSF3R tyrosines by using a tyrosine $(\mathrm{Y})$ less $(\mathrm{m} 0)$ and single tyrosine addback CSF3R mutants (mA, mB, mC and $\mathrm{mD}$ ) containing respectively $\mathrm{Y} 704, \mathrm{Y} 729, \mathrm{Y} 744$ and Y764 (Figure 1D). The presence of STAT3 docking sites Y704 or Y744 (mA and mC) resulted in an approximately 1.5 times higher and a prolonged induction of $D u b 2 A$ relative to $\mathrm{m} 0$. The presence of SOCS3 recruitment site $Y 729(\mathrm{mB})$ decreased the induction of $D u b 2 A$ expression approximately 2-fold compared to $\mathrm{m} 0$. The presence of Y764 (mD), involved in the activation of the Ras/Raf/Erk pathway [37], resulted in only a moderate (1.5-fold) increase of Dub2A expression, suggesting that, in contrast to IL3-induced expression of Dub1, Erk is not essential for CSF3-induced Dub2A expression (Figure 1D). To further investigate which signalling pathways might be involved in this process, we used a panel of chemical inhibitors, including MEK [MAPK (mitogen activated/protein kinase)/ERK (extracellular-signalregulated kinase) kinase] inhibitor U0126, Src tyrosine kinase inhibitor PP2, Akt inhibitor IV, p38 MAPK inhibitor SB203580, JAK inhibitor I, PI-3K (phosphoinositide-3 kinase) inhibitor LY294002 and JNK inhibitor VIII. JAK inhibitor I completely and MEK inhibitor U0126 partly abolished $D u b 2 A$ upregulation. None of the other compounds showed significant inhibitory effects (Figure 1E).

\section{DUB2 reduces ubiquitination and enhances protein stability of activated CSF3R in the retrograde pathway}

Next, we asked if DUB2 affects the CSF3R ubiquitination status and whether this has an impact on CSF3R degradation. Ectopic expression of DUB2 reduced ubiquitination levels of activated wt CSF3Rs (Figure 2A and 2B). CSF3R mutant K5R, which lacks all cytoplasmic lysines and consequently escapes lysosomal degradation [7], was taken along as a relative measure of CSF3R protein stability. Importantly, although the expression of UBPY, AMSH and DUB2 all resulted in reduced CSF3R ubiquitination levels, only DUB2 increased the stability of the wt CSF3R, comparable to the levels of K5R (Figure 2A). In contrast, ectopic expression of AMSH led to increased degradation of the CSF3R, whereas UBPY did not influence CSF3R stability (Figure 2A and 2B). 


\section{DUB2 does not affect total levels of nonactivated CSF3R}

Subsequently, we wondered if DUBs also influence steady state CSF3R levels. Expression of DUB2 did not elevate the total CSF3R pool, implicating that DUB2-mediated deubiquitination and increased protein stability is specifically associated with the retrograde endocytotic pathway (Figure 2C). Strikingly, expression of AMSH resulted in increased degradation of the total pool of CSF3R, suggesting that AMSH exerts a negative role in CSF3R processing in the biosynthetic pathway. On the other hand, UBPY, like DUB2, did not affect total CSF3R levels.

\section{DUB2 promotes retention of activated CSF3R in early endosomes}

Previously, we showed that ubiquitination of CSF3R is not required for CSF3R internalization but that ubiquitination of a juxtamembrane lysine residue (Lys-632) is crucial for CSF3R routing from early endosomes towards late endosomes and lysosomes, thereby causing the degradation of the activated CSF3R [7, 43]. Because DUB2 enhances CSF3R protein stability we asked whether DUB2 directly interferes with lysosomal routing by controlling the ubiquitination status of Lys-632. To this end, we employed a mutant, mKA, that retains Lys-632 but lacks the other conserved cytoplasmic lysine residues [7]. DUB2 clearly increased the stability of the mKA CSF3R, providing additional evidence for a role of DUB2 in counteracting lysosomal degradation of CSF3R (Figure 3A and 3B) and further supporting the crucial involvement of Lys-632 and its ubiquitination status in balancing this process.

Next, we studied the effects of DUB2 on the routing dynamics of CSF3R by confocal microscopy. At low endogenous DUB levels, ligand-activated CSF3Rs resided in lysosomes 2 hrs after CSF3 stimulation and were no longer detectable in early endosomes (Figure 3C, upper panel). In contrast, in cells in which DUB2 was ectopically expressed, CSF3R accumulated in early endosomes 2 hrs post CSF3 stimulation (Figure 3C, second panel). Notably, ectopic expression of UBPY or AMSH did not result in increased accumulation of CSF3R in the early endosome compartment, clearly suggesting that these enzymes deubiquitinate CSF3R at a different stage in the retrograde pathway (Figure 3C, third and fourth panel).

\section{DUB2 prolongs CSF3-induced STAT5 phosphorylation}

Accumulation of lysine-deficient mutants of CSF3R in early endosomes causes sustained activation of STAT5 upon removal of CSF3 [7]. To investigate whether upregulation of DUB2 has a similar effect on STAT5 activation, Ba/F3ß/tTA-DUB2 cells stably expressing wt CSF3R were cultured for $60 \mathrm{hrs}$ with Dox to inhibit and without Dox to induce DUB2 expression. Expression of DUB2 resulted in prolonged STAT5 phosphorylation after CSF3 withdrawal (Figure 4A and 4B). Pull down experiments with bio-CSF3 showed that this was associated with reduced ubiquitination of the CSF3R (Figure 4C and 4D).

\section{DISCUSSION}

We have identified the USP family member DUB2A as a major negative regulator of CSF3R ubiquitination, thereby controlling intracellular routing and signalling properties of CSF3R. Among the 4 homologous members of the murine DUB family, only DUB2A was specifically up-regulated in myeloid cells in response to CSF3 stimulation. DUB2A reduced the ubiquitination of the juxtamembrane lysine 632, which was previously shown to be a target for ubiquitination by the E3 ligase SOCS3 [7]. 
It is currently unknown why DUB2A, but not other family members, is specifically upregulated in myeloid cells and which signalling pathways and transcriptional regulators are important for its expression. Sequence alignment of the 5' transcription enhancer domains of $D u b 1$ and $D u b 2 A$ showed that these regions contain comparable transcription factor binding sites, including GATA, ETS, AP1 and CBF sites [23]. Potentially, differential accessibility of promoter/enhancer regions, e.g., due to chromatin structure, could explain why only Dub2A and not Dub1, Dub1A or Dub2 is transcribed in myeloid 32D cells in response to CSF3, but this needs further study. Concerning the signalling mechanisms involved, it became clear that activation of JAKs is crucial for CSF3-induced Dub2A expression. In contrast, no evidence for a major involvement of other signalling pathways activated by CSF3, e.g., Ras/Raf/Mek1, PI3K/Akt, p38MAPK or Jnk pathways was obtained, either by applying specific inhibitors or from experiments with selective tyrosine mutants of CSF3R known to play a major role in the activation of STAT3 or MAP kinases [37, 39-40, 44].

Although DUB2, UBPY and AMSH all reduced ubiquitination of activated CSF3Rs, only DUB2 enhanced the stability of the CSF3R, through promoting its accumulation in early endosomes. For UBPY, this fits well with its proposed function to retrieve ubiquitin from targeted receptors before they go into MVB [45]. In literature, there are opposing reports about the role of AMSH in EGFR degradation. Whereas one study shows that AMSH is important for EGFR degradation after retrograde transport [16] another demonstrates that AMSH silencing enhances EGFR degradation [8]. We showed that AMSH strongly diminishes the steady-state CSF3R levels independent of receptor activation and internalization. The finding that decreased receptor levels were pulled down with bio-CSF3, might thus be entirely due to reduced membrane expression of CSF3R, rather than elevated degradation after retrograde transport. At this point we cannot discriminate if DUB2 directly or indirectly reduces the ubiquitation of the CSF3R. DUB2 may deubiquitinate members of the endocytotic machinery and thereby influencing the routing and the ubiquitination status of the CSF3 receptor. This is not without precedent because it is known that DUB1 deubiquitinates dynein heavy chain [32]. The dynein heavy chain family, which has two major classes of axonemal and cytoplasmic proteins, mainly associates with microtubule complex and is involved in the regulation of the movement of organelles and vesicles along microtubules [46]. Dynein is known to be associated with the movement of late endosomes and also for receptor sorting and morphogenesis of early endosomes [47-48].

In conclusion, the data presented here fit into a model in which ubiquitination of activated CSF3R is dynamically controlled by the E3 ligase activity of SOCS3, a direct target of STAT3 [44], and the Ub specific protease activity of DUB2A (Figure 5). Given that receptor ubiquitination has been implicated in the endocytotic routing of numerous membrane receptors involving a variety of E3 ligases [43, 49-52], it is now worth-while to investigate whether and which DUBs are able to delay the lysosomal routing of different growth factor receptor systems. Conceivably, this will give important insights into how these receptor activities are controlled under physiological conditions. Moreover, in view of the increasing association of ubiquitin-mediated pathways with various pathological conditions, the role of DUBs in diseases linked with growth factor receptor dysfunction deserves further study.

\section{ACKNOWLEDGMENTS}

This work was supported by the Dutch Cancer Society, KWF kankerbestrijding (www.KWFkankerbestrijding.nl ). We are grateful to Dr. James A. Johnston (Belfast, UK) for Ba/F3 $\beta /$ tTA DUB2, pMEF-DUB2 and DUB2 antibodies and to Dr. Sylvie Urbé (Liverpool, UK) for providing pEGFP-C1-AMSH and pEGFP-C1-UBPY constructs.

\section{FUNDING}

This work was supported by the Dutch Cancer Society, KWF kankerbestrijding [grant number EMCR 2006-3585]. 


\section{REFERENCES}

1. Zwang, Y. and Yarden, Y. (2009) Systems biology of growth factor-induced receptor endocytosis. Traffic 10, 349-363

2. Raiborg, C. and Stenmark, H. (2009) The ESCRT machinery in endosomal sorting of ubiquitylated membrane proteins. Nature 458, 445-452

3. Lieschke, G.J., Grail, D., Hodgson, G., Metcalf, D., Stanley, E., Cheers, C., Fowler, K.J., Basu, S., Zhan, Y.F., and Dunn, A.R. (1994) Mice lacking granulocyte colony-stimulating factor have chronic neutropenia, granulocyte and macrophage progenitor cell deficiency, and impaired neutrophil mobilization. Blood 84, 1737-1746

4. Hortner, M., Nielsch, U., Mayr, L.M., Johnston, J.A., Heinrich, P.C., and Haan, S. (2002) Suppressor of cytokine signaling-3 is recruited to the activated granulocyte-colony stimulating factor receptor and modulates its signal transduction. J. Immunol. 169, 1219-1227

5. Tian, S.S., Lamb, P., Seidel, H.M., Stein, R.B., and Rosen, J. (1994) Rapid activation of the STAT3 transcription factor by granulocyte colonystimulating factor. Blood 84, 1760-1764

6. Babon, J.J., Sabo, J.K., Soetopo, A., Yao, S., Bailey, M.F., Zhang, J.G., Nicola, N.A., and Norton, R.S. (2008) The SOCS box domain of SOCS3: structure and interaction with the elonginBC-cullin5 ubiquitin ligase. J. Mol. Biol. 381, 928-940

7. Irandoust, M.I., Aarts, L.H., Roovers, O., Gits, J., Erkeland, S.J., and Touw, I.P. (2007) Suppressor of cytokine signaling 3 controls lysosomal routing of G-CSF receptor. Embo J. 26, 1782-1793

8. McCullough, J., Clague, M.J., and Urbe, S. (2004) AMSH is an endosomeassociated ubiquitin isopeptidase. J. Cell Biol. 166, 487-492

9. Kato, M., Miyazawa, K., and Kitamura, N. (2000) A deubiquitinating enzyme UBPY interacts with the Src homology 3 domain of Hrs-binding protein via a novel binding motif PX(V/I)(D/N)RXXKP. J. Biol. Chem. 275, 37481-37487

10. Agromayor, M. and Martin-Serrano, J. (2006) Interaction of AMSH with ESCRT-III and deubiquitination of endosomal cargo. J. Biol. Chem. 281, 23083-23091

11. Kaneko, T., Kumasaka, T., Ganbe, T., Sato, T., Miyazawa, K., Kitamura, N., and Tanaka, N. (2003) Structural insight into modest binding of a non-PXXP ligand to the signal transducing adaptor molecule-2 Src homology 3 domain. J. Biol. Chem. 278, 48162-48168

12. Row, P.E., Liu, H., Hayes, S., Welchman, R., Charalabous, P., Hofmann, K., Clague, M.J., Sanderson, C.M., and Urbe, S. (2007) The MIT domain of UBPY constitutes a CHMP binding and endosomal localization signal required for efficient epidermal growth factor receptor degradation. J. Biol. Chem. 282, 30929-30937

13. Tanaka, N., Kaneko, K., Asao, H., Kasai, H., Endo, Y., Fujita, T., Takeshita, T., and Sugamura, K. (1999) Possible involvement of a novel STAMassociated molecule "AMSH" in intracellular signal transduction mediated by cytokines. J. Biol. Chem. 274, 19129-19135

14. Hasdemir, B., Murphy, J.E., Cottrell, G.S., and Bunnett, N.W. (2009) Endosomal deubiquitinating enzymes control ubiquitination and downregulation of protease-activated receptor 2. J. Biol. Chem. 284, 28453-28466 
15. Hislop, J.N., Henry, A.G., Marchese, A., and von Zastrow, M. (2009) Ubiquitination regulates proteolytic processing of $\mathrm{G}$ protein-coupled receptors after their sorting to lysosomes. J. Biol. Chem. 284, 19361-19370

16. Ma, Y.M., Boucrot, E., Villen, J., Affar el, B., Gygi, S.P., Gottlinger, H.G., and Kirchhausen, T. (2007) Targeting of AMSH to endosomes is required for epidermal growth factor receptor degradation. J. Biol. Chem. 282, 9805-9812

17. Sierra, M.I., Wright, M.H., and Nash, P.D. (2010) AMSH interacts with ESCRT-0 to regulate the stability and trafficking of CXCR4. J. Biol. Chem. 285, 13990-14004

18. Row, P.E., Prior, I.A., McCullough, J., Clague, M.J., and Urbe, S. (2006) The ubiquitin isopeptidase UBPY regulates endosomal ubiquitin dynamics and is essential for receptor down-regulation. J. Biol. Chem. 281, 12618-12624

19. Niendorf, S., Oksche, A., Kisser, A., Lohler, J., Prinz, M., Schorle, H., Feller, S., Lewitzky, M., Horak, I., and Knobeloch, K.P. (2007) Essential role of ubiquitin-specific protease 8 for receptor tyrosine kinase stability and endocytic trafficking in vivo. Mol. Cell Biol. 27, 5029-5039

20. Mizuno, E., Kobayashi, K., Yamamoto, A., Kitamura, N., and Komada, M. (2006) A deubiquitinating enzyme UBPY regulates the level of protein ubiquitination on endosomes. Traffic 7, 1017-1031

21. Alwan, H.A. and van Leeuwen, J.E. (2007) UBPY-mediated epidermal growth factor receptor (EGFR) de-ubiquitination promotes EGFR degradation. J. Biol. Chem. 282, 1658-1669

22. Naviglio, S., Mattecucci, C., Matoskova, B., Nagase, T., Nomura, N., Di Fiore, P.P., and Draetta, G.F. (1998) UBPY: a growth-regulated human ubiquitin isopeptidase. Embo J. 17, 3241-3250

23. Baek, K.H., Mondoux, M.A., Jaster, R., Fire-Levin, E., and D'Andrea, A.D. (2001) DUB-2A, a new member of the DUB subfamily of hematopoietic deubiquitinating enzymes. Blood 98, 636-642

24. Zhu, Y., Lambert, K., Corless, C., Copeland, N.G., Gilbert, D.J., Jenkins, N.A., and D'Andrea, A.D. (1997) DUB-2 is a member of a novel family of cytokine-inducible deubiquitinating enzymes. J. Biol. Chem. 272, 51-57

25. Zhu, Y., Pless, M., Inhorn, R., Mathey-Prevot, B., and D'Andrea, A.D. (1996) The murine DUB-1 gene is specifically induced by the betac subunit of interleukin-3 receptor. Mol. Cell Biol. 16, 4808-4817

26. Baek, K.H., Kim, M.S., Kim, Y.S., Shin, J.M., and Choi, H.K. (2004) DUB$1 \mathrm{~A}$, a novel deubiquitinating enzyme subfamily member, is polyubiquitinated and cytokine-inducible in B-lymphocytes. J. Biol. Chem. 279, 2368-2376

27. Zhu, Y., Carroll, M., Papa, F.R., Hochstrasser, M., and D'Andrea, A.D. (1996) DUB-1, a deubiquitinating enzyme with growth-suppressing activity. Proc. Natl. Acad. Sci. U.S.A. 93, 3275-3279

28. Jaster, R., Zhu, Y., Pless, M., Bhattacharya, S., Mathey-Prevot, B., and D'Andrea, A.D. (1997) JAK2 is required for induction of the murine DUB-1 gene. Mol. Cell Biol. 17, 3364-3372

29. Jaster, R., Baek, K.H., and D'Andrea, A.D. (1999) Analysis of cis-acting sequences and trans-acting factors regulating the interleukin-3 response element of the DUB-1 gene. Biochim. Biophys. Acta 1446, 308-316

30. Migone, T.S., Humbert, M., Rascle, A., Sanden, D., D'Andrea, A., and Johnston, J.A. (2001) The deubiquitinating enzyme DUB-2 prolongs cytokineinduced signal transducers and activators of transcription activation and suppresses apoptosis following cytokine withdrawal. Blood 98, 1935-1941 
31. Baek, K.H., Kim, Y.S., Lee, H.J., and Kang, I. (2004) Essential regions of deubiquitinating enzyme activity and enhancer function for DUB-2A expressed in T-lymphocytes. Arch. Biochem. Biophys. 430, 191-197

32. Lee, M.Y., Ajjappala, B.S., Kim, M.S., Oh, Y.K., and Baek, K.H. (2008) DUB-1, a fate determinant of dynein heavy chain in B-lymphocytes, is regulated by the ubiquitin-proteasome pathway. J. Cell. Biochem. 105, 14201429

33. Burrows, J.F., McGrattan, M.J., Rascle, A., Humbert, M., Baek, K.H., and Johnston, J.A. (2004) DUB-3, a cytokine-inducible deubiquitinating enzyme that blocks proliferation. J. Biol. Chem. 279, 13993-14000

34. Okada, T., Gondo, Y., Goto, J., Kanazawa, I., Hadano, S., and Ikeda, J.E. (2002) Unstable transmission of the RS447 human megasatellite tandem repetitive sequence that contains the USP17 deubiquitinating enzyme gene. Hum. Genet. 110, 302-313

35. Burrows, J.F., Scott, C.J., and Johnston, J.A. (2010) The DUB/USP17 deubiquitinating enzymes: a gene family within a tandemly repeated sequence, is also embedded within the copy number variable beta-defensin cluster. BMC Genomics 11, 250

36. Burrows, J.F., McGrattan, M.J., and Johnston, J.A. (2005) The DUB/USP17 deubiquitinating enzymes, a multigene family within a tandemly repeated sequence. Genomics 85, 524-529

37. de Koning, J.P., Soede-Bobok, A.A., Schelen, A.M., Smith, L., van Leeuwen, D., Santini, V., Burgering, B.M., Bos, J.L., Lowenberg, B., and Touw, I.P. (1998) Proliferation signaling and activation of Shc, p21Ras, and Myc via tyrosine 764 of human granulocyte colony-stimulating factor receptor. Blood 91, 1924-1933

38. Aarts, L.H., Roovers, O., Ward, A.C., and Touw, I.P. (2004) Receptor activation and 2 distinct $\mathrm{COOH}$-terminal motifs control G-CSF receptor distribution and internalization kinetics. Blood 103, 571-579

39. Ward, A.C., Hermans, M.H., Smith, L., van Aesch, Y.M., Schelen, A.M., Antonissen, C., and Touw, I.P. (1999) Tyrosine-dependent and -independent mechanisms of STAT3 activation by the human granulocyte colonystimulating factor (G-CSF) receptor are differentially utilized depending on GCSF concentration. Blood 93, 113-124

40. Hermans, M.H., van de Geijn, G.J., Antonissen, C., Gits, J., van Leeuwen, D., Ward, A.C., and Touw, I.P. (2003) Signaling mechanisms coupled to tyrosines in the granulocyte colony-stimulating factor receptor orchestrate G-CSFinduced expansion of myeloid progenitor cells. Blood 101, 2584-2590

41. van de Geijn, G.J., Gits, J., and Touw, I.P. (2004) Distinct activities of suppressor of cytokine signaling (SOCS) proteins and involvement of the SOCS box in controlling G-CSF signaling. J. Leukoc. Biol. 76, 237-244

42. Hermans, M.H., Antonissen, C., Ward, A.C., Mayen, A.E., Ploemacher, R.E., and Touw, I.P. (1999) Sustained receptor activation and hyperproliferation in response to granulocyte colony-stimulating factor (G-CSF) in mice with a severe congenital neutropenia/acute myeloid leukemia-derived mutation in the G-CSF receptor gene. J. Exp. Med. 189, 683-692

43. Wolfler, A., Irandoust, M., Meenhuis, A., Gits, J., Roovers, O., and Touw, I.P. (2009) Site-specific ubiquitination determines lysosomal sorting and signal attenuation of the granulocyte colony-stimulating factor receptor. Traffic $\mathbf{1 0}$, 1168-1179 
44. Zhuang, D., Qiu, Y., Haque, S.J., and Dong, F. (2005) Tyrosine 729 of the GCSF receptor controls the duration of receptor signaling: involvement of SOCS3 and SOCS1. J. Leukoc. Biol. 78, 1008-1015

45. Clague, M.J. and Urbe, S. (2006) Endocytosis: the DUB version. Trends Cell Biol. 16, 551-559

46. Shima, T., Kon, T., Imamula, K., Ohkura, R., and Sutoh, K. (2006) Two modes of microtubule sliding driven by cytoplasmic dynein. Proc. Natl. Acad. Sci. U.S.A. 103, 17736-17740

47. Bananis, E., Nath, S., Gordon, K., Satir, P., Stockert, R.J., Murray, J.W., and Wolkoff, A.W. (2004) Microtubule-dependent movement of late endocytic vesicles in vitro: requirements for Dynein and Kinesin. Mol. Biol. Cell 15, 3688-3697

48. Driskell, O.J., Mironov, A., Allan, V.J., and Woodman, P.G. (2007) Dynein is required for receptor sorting and the morphogenesis of early endosomes. Nat. Cell Biol. 9, 113-120

49. Fernandez-Sanchez, E., Martinez-Villarreal, J., Gimenez, C., and Zafra, F. (2009) Constitutive and regulated endocytosis of the glycine transporter GLYT1b is controlled by ubiquitination. J. Biol Chem. 284, 19482-19492

50. Goh, L.K., Huang, F., Kim, W., Gygi, S., and Sorkin, A. (2010) Multiple mechanisms collectively regulate clathrin-mediated endocytosis of the epidermal growth factor receptor. J. Cell Biol. 189, 871-883

51. Marmor, M.D. and Yarden, Y. (2004) Role of protein ubiquitylation in regulating endocytosis of receptor tyrosine kinases. Oncogene 23, 2057-2070

52. Molfetta, R., Gasparrini, F., Santoni, A., and Paolini, R. (2010) Ubiquitination and endocytosis of the high affinity receptor for IgE. Mol. Immunol. 47, 24272434 


\section{FIGURE LEGENDS}

Figure 1 Myeloid 32D cells stably expressing wt CSF3R or receptor tyrosine mutants transiently induce Dub2A mRNA after CSF3 stimulation. 32D cells were deprived of growthfactors for $4 \mathrm{hrs}(-)$ and stimulated with CSF3 for the indicated time points (A) Dub2A, Amsh and Ubpy mRNA expression levels in wt CSF3R expressing cells were determined using RTPCR and normalized using the ribonuclease inhibitor (RI). Dub levels are shown relative to the growth-factor deprived cells. Data represent mean values of three independent experiments \pm standard deviation (SD). (B) Socs3 mRNA levels in wt CSF3R were determined as described in A. (C) DUB2A protein detected using DUB2 antibody on Western blot of total cell lysates of the 32D cells expressing wt CSF3R stimulated with CSF3 for the indicated time points. (D) Dub2A mRNA levels in wt, tyrosine null (m0) and single tyrosine add-back CSF3Rs (mA-mD) were determined as in A. Dub2A levels are shown relative to growth-factor deprived cells. (E) Dub2A induction was assessed in the presence of various kinase inhibitors (see text for details) as described in A. Data represent the mean of three independent experiments \pm SD.

Figure 2 Overexpression of DUB2 or UBPY influences ubiquitination level of wt CSF3R. (A) $293 \mathrm{~T}$ cells were transfected with the indicated constructs together with Flag-Ub and after $48 \mathrm{hrs}$ the cells were incubated with biotinylated CSF3 (Bio-CSF3) for $30 \mathrm{~min}$ at $37^{\circ} \mathrm{C}$. Ligand-bound receptors were precipitated using streptavidin-coated beads and analyzed by Western blotting using Flag and CSF3R antibodies. To confirm the presence of different deubiquitinating enzymes, total cell lysate (TCL) blot was stained using GFP and DUB2 antibodies. TCL blot was also stained for ACTIN as loading control. (B) Quantification of three experiments as shown in A. The mean amounts of Ub in the CSF3R immunoprecipitates were normalized to total receptor levels and expressed as a percentage of the ubiquitination status of wt CSF3R $\pm \mathrm{SD}$. (C) 239T cells were transfected with indicated constructs. After 48h TCL was taken. The blot was stained for CSF3R, GFP, DUB2 and ACTIN as a loading control. Wt: CSF3R wild type; K5R: CSF3R mutant lacking conserved cytosolic lysines.

Figure 3 Enhanced levels of DUB2 cause CSF3R accumulation in early endosomes. (A and B) Deubiquitination of Lys-632 by DUB2. Experiment performed as described for Figure 2A and 2B. (C) HeLa stably expressing wt CSF3R were transfected with ev (first panel), DUB2 (second panel), GFP-UBPY (third panel) or GFP-AMSH (fourth panel). After $48 \mathrm{hrs}$ the cells were incubated with CSF3 and CSF3R antibody for $10 \mathrm{~min}$ at RT, washed and put at $37^{\circ} \mathrm{C}$ for $2 \mathrm{hrs}$ to stimulate lysosomal routing. Subsequently cells were fixed and stained for EEA1 and CSF3R in combination with or without DUB2. Scale Bar, $10 \mu \mathrm{m}$.

Figure 4 Prolonged STAT5 signalling of wt CSF3R deubiquitinated by DUB2.

$\mathrm{Ba} / \mathrm{F} 3 \beta / \mathrm{tTA}$ DUB2 cells stably expressing wt CSF3R were grown in the presence or absence of dox for $60 \mathrm{hrs}$. (A) Cells were cytokine and serum deprived for $4 \mathrm{hrs}$, stimulated for 10 min with CSF3 at $37^{\circ} \mathrm{C}$. The CSF3 was washed away and cells were kept in medium without factor at $37^{\circ} \mathrm{C}$ for the indicated time points. Down regulation of STAT5 phosphorylation was monitored using Western Blot. The blot was stained for total STAT5 as loading control.

(B) Quantification of three experiments as shown in A. The mean amounts of pSTAT5 were normalized to total levels and expressed as a percentage of maximum pSTAT5 activation \pm SEM. (C) Cells were pre-incubated with the lysosomal inhibitor bafilomycin and stimulated with Bio-CSF3 for the indicated time periods. CSF3R were precipitated using streptavidin beads and analyzed by Western blotting for CSF3R and endogenous ubiquitin using P4D1 antibody. TCL blot is shown to confirm upregulation of DUB2 in the absence of dox and stained for actin as a loading control. (D) Quantification of 3 independent experiments shown in $\mathrm{B}$. The mean amounts of $\mathrm{Ub}$ in the CSF3R immunoprecipitates were normalized to total receptor levels and expressed as a percentage \pm SD of ubiquitination status of wt CSF3R after 60 min Bio-CSF3 stimulation in the absence of DUB2 (set at 100\%). 
Figure 5 Model of SOCS3 and DUB2A controlled CSF3R routing and signal termination. Following CSF3 stimulation STAT3 is activated which induces SOCS3. SOCS3 is subsequently recruited to Y729 of the CSF3R and forms an E3 ligase complex via its SOCS box that ubiquitinates Lys-632 of the CSF3R (negative feedback loop). This causes CSF3R routing from early endosomes to lysosomes where the receptor is degraded. DUB2A is in addition upregulated by CSF3 via an unknown mechanism which involves activation of JAKs. DUB2A deubiquitinates Lys-632 of the CSF3R, thereby counteracting the SOCS3 ligase complex and inhibiting lysosomal routing (positive feedback loop). 


\section{Figure 1}

A

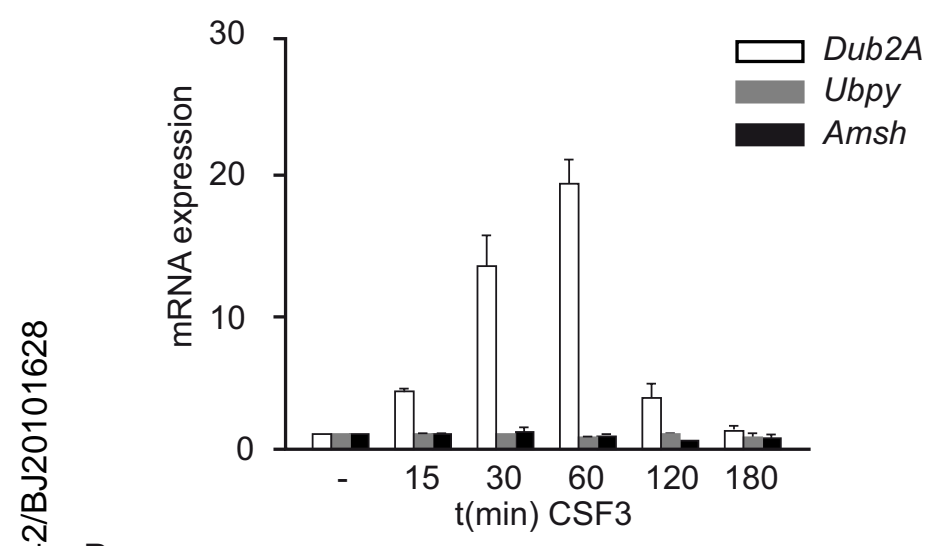

D

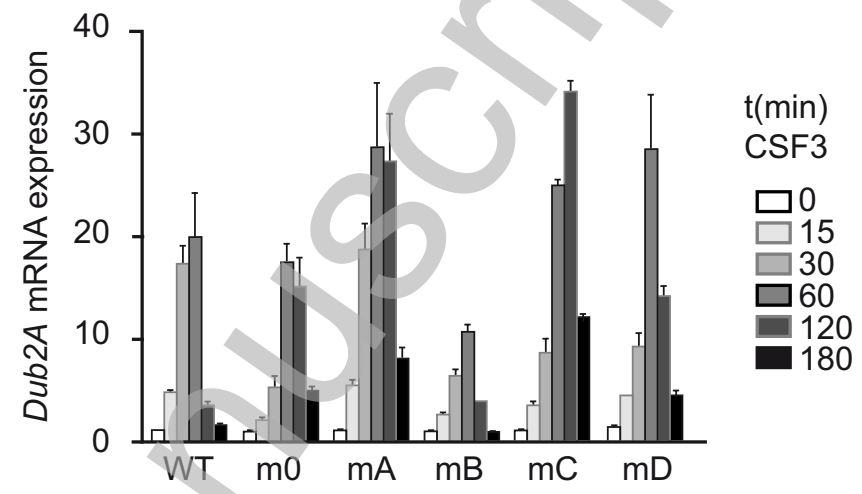

$B$

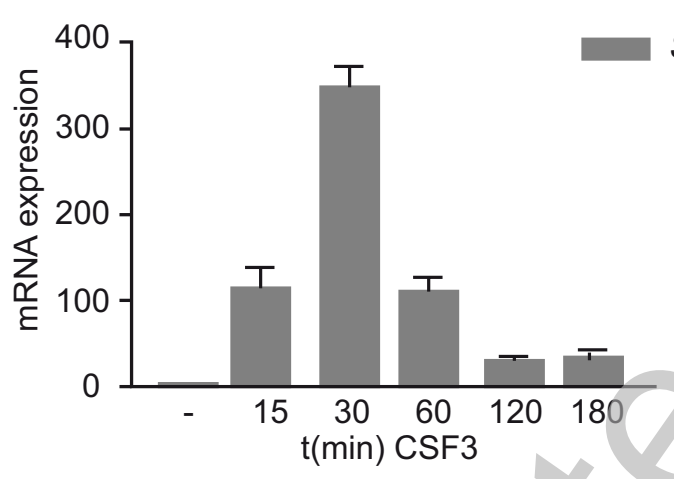

E

Socs3

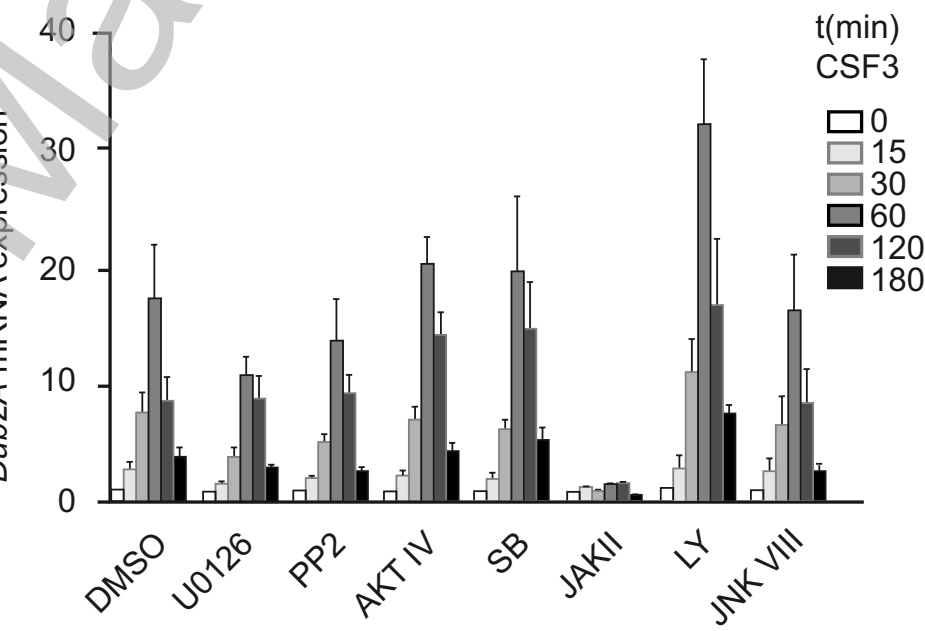

C

岌

$\begin{array}{lllllll}t(\min ) \text { CSF3 } & 0 & 15 & 30 & 60 & 120 & 180\end{array}$

DUB2A

ACTIN

Licenced copy. Copying is not permitted, except with prior permission and as allowed by law.

(C) 2010 The Authors Journal compilation (c) 2010 Portland Press Limited 
Figure 2

A

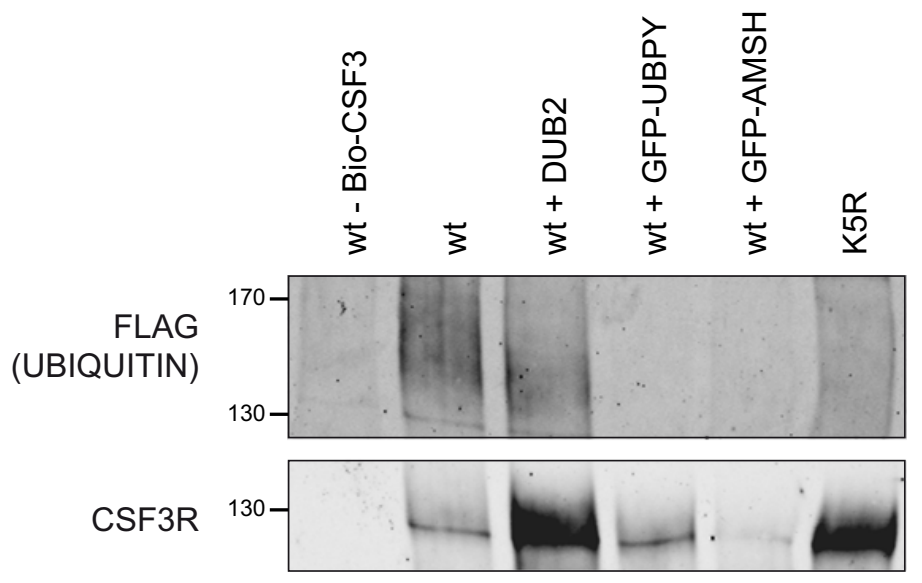

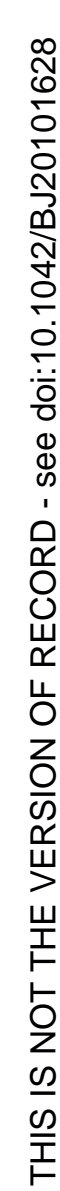

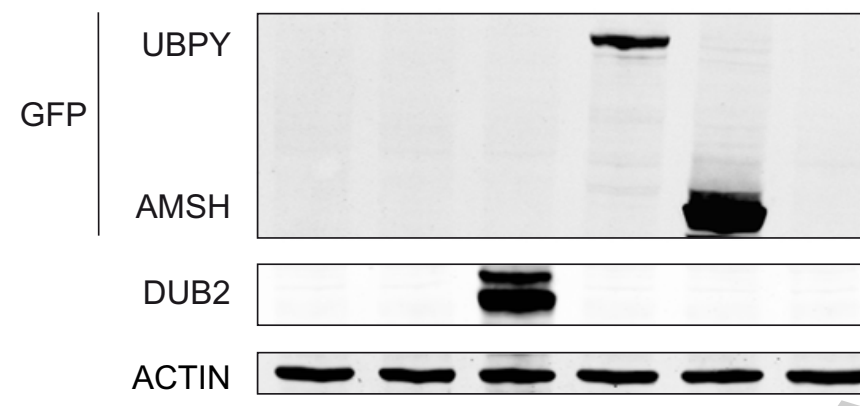

Bio-CSF3

pulldown

TCL

B

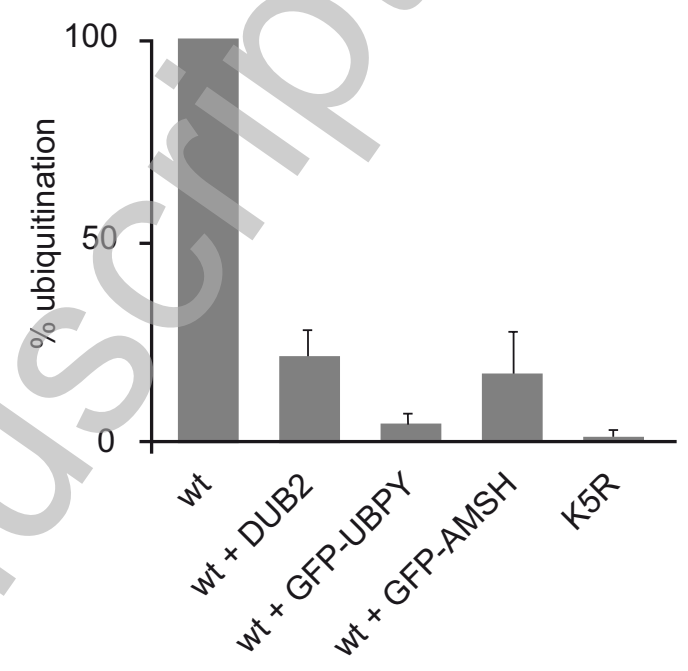

C

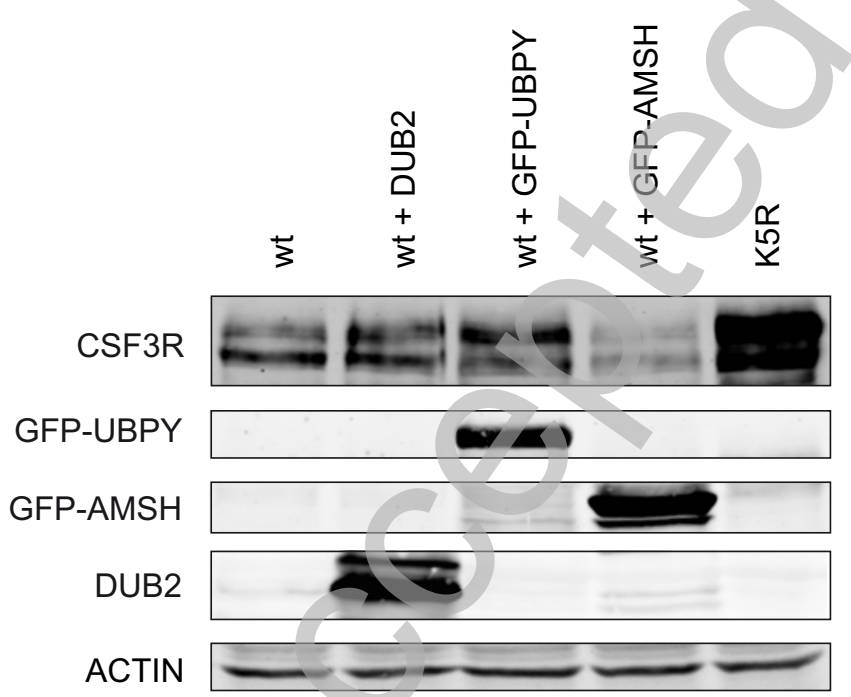


B) Biochemical Journal Immediate Publication. Published on 15 Dec 2010 as manuscript BJ20101628

Figure 3

A
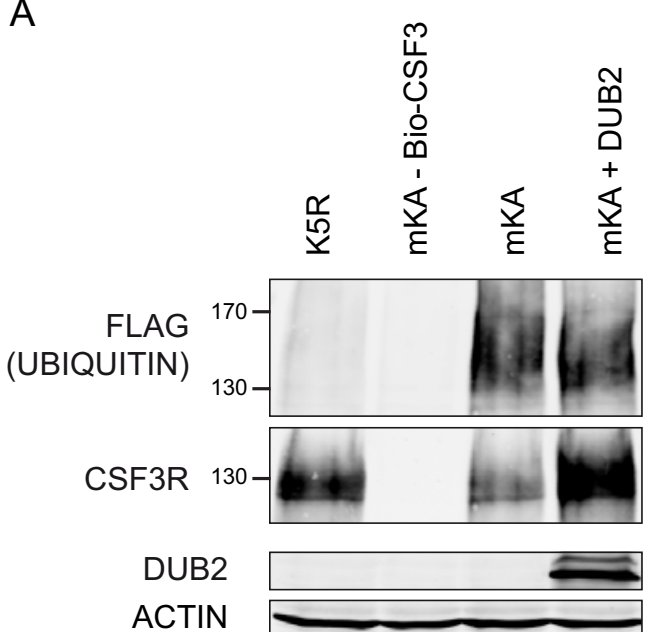

Bio-CSF3

pulldown

TCL
B

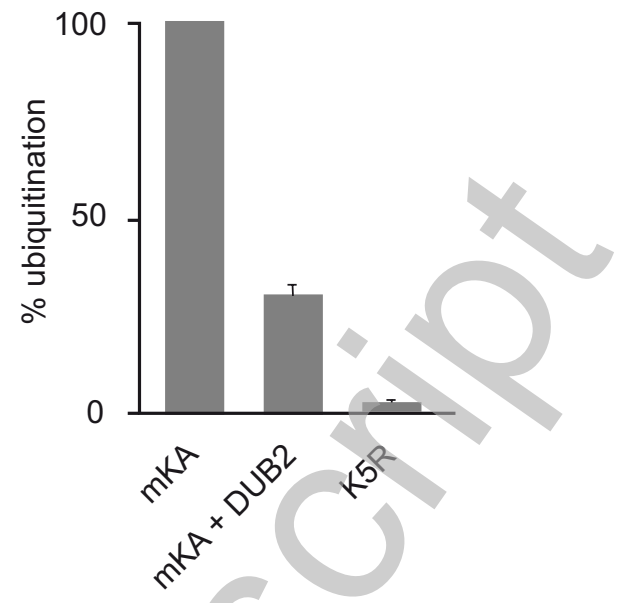

C
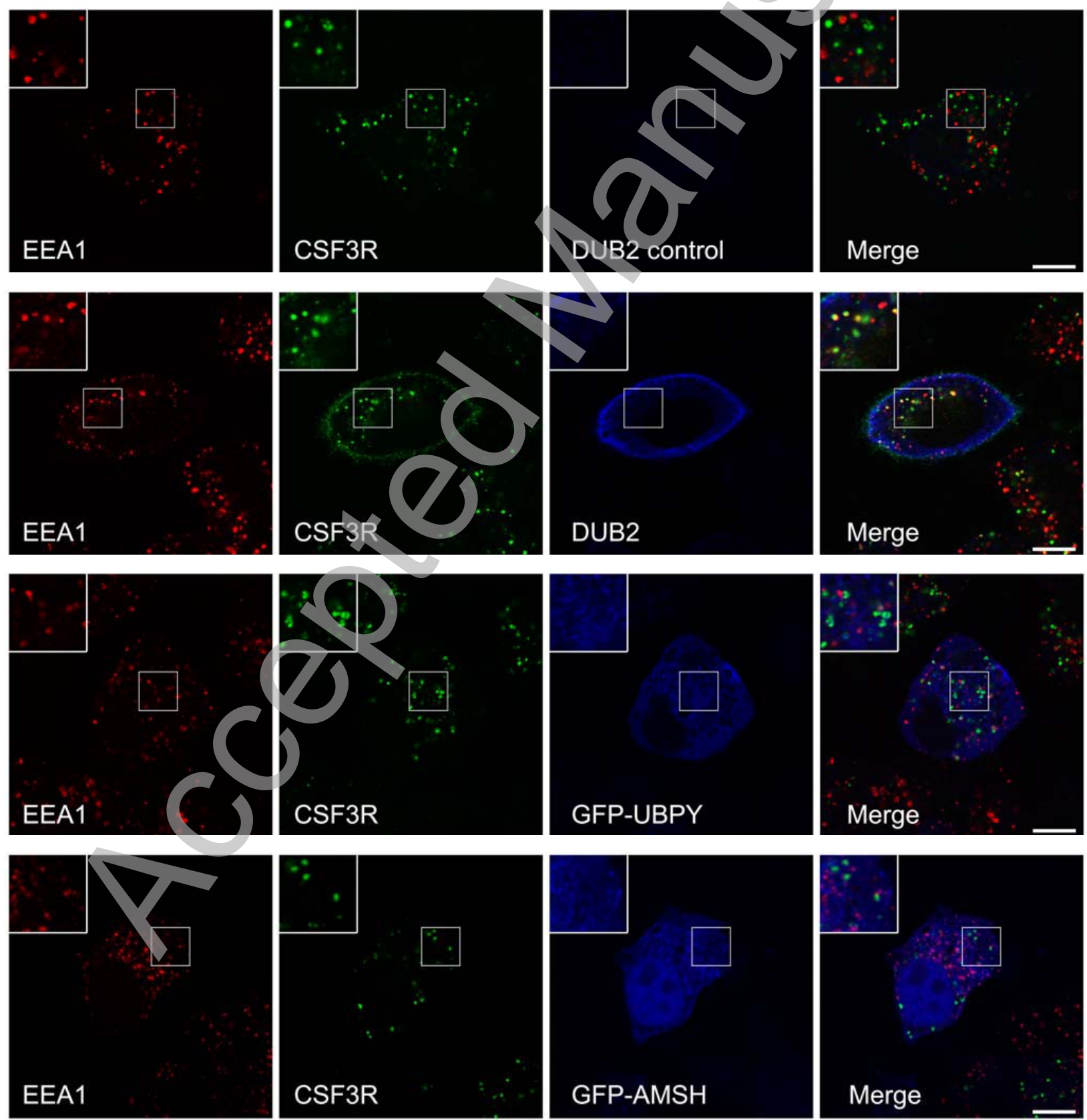

Licenced copy. Copying is not permitted, except with prior permission and as allowed by law. (C) 2010 The Authors Journal compilation (c) 2010 Portland Press Limited 
Figure 4

A

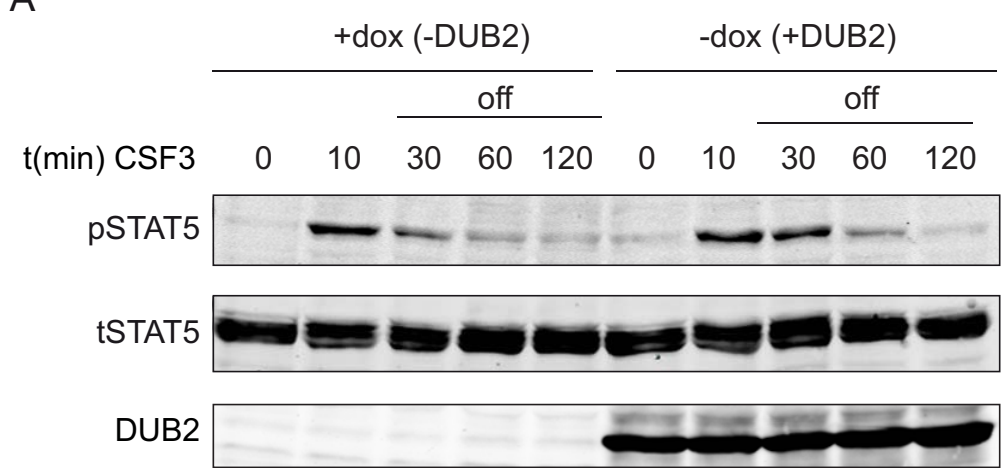

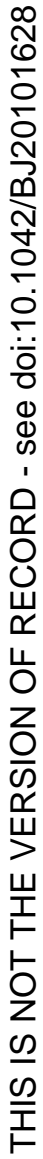

C

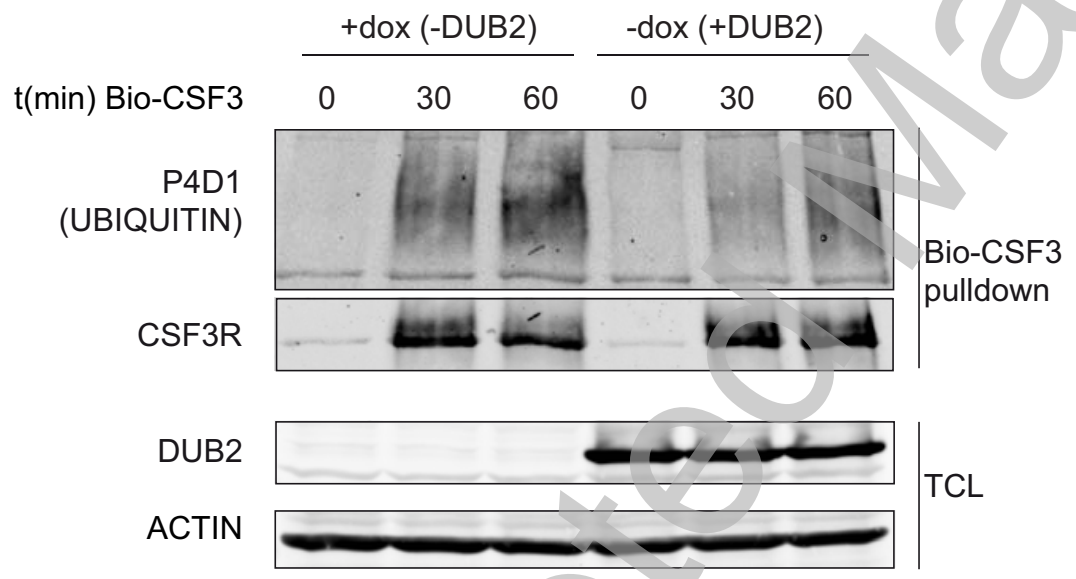

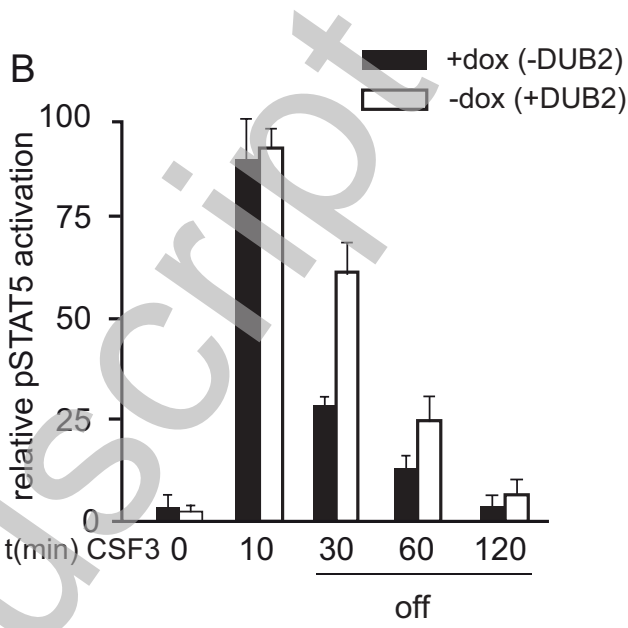

D

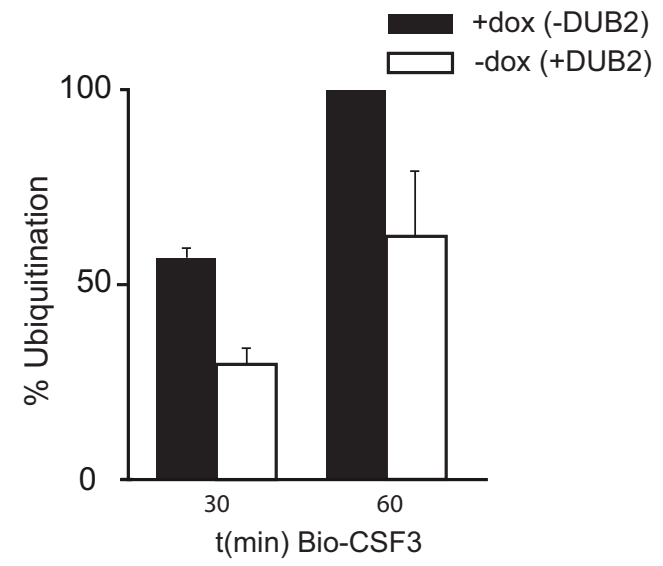




\section{B Biochemical Journal Immediate Publication. Published on 15 Dec 2010 as manuscript BJ20101628}

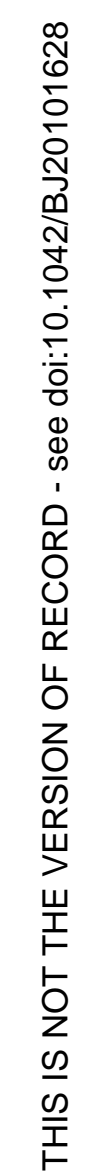

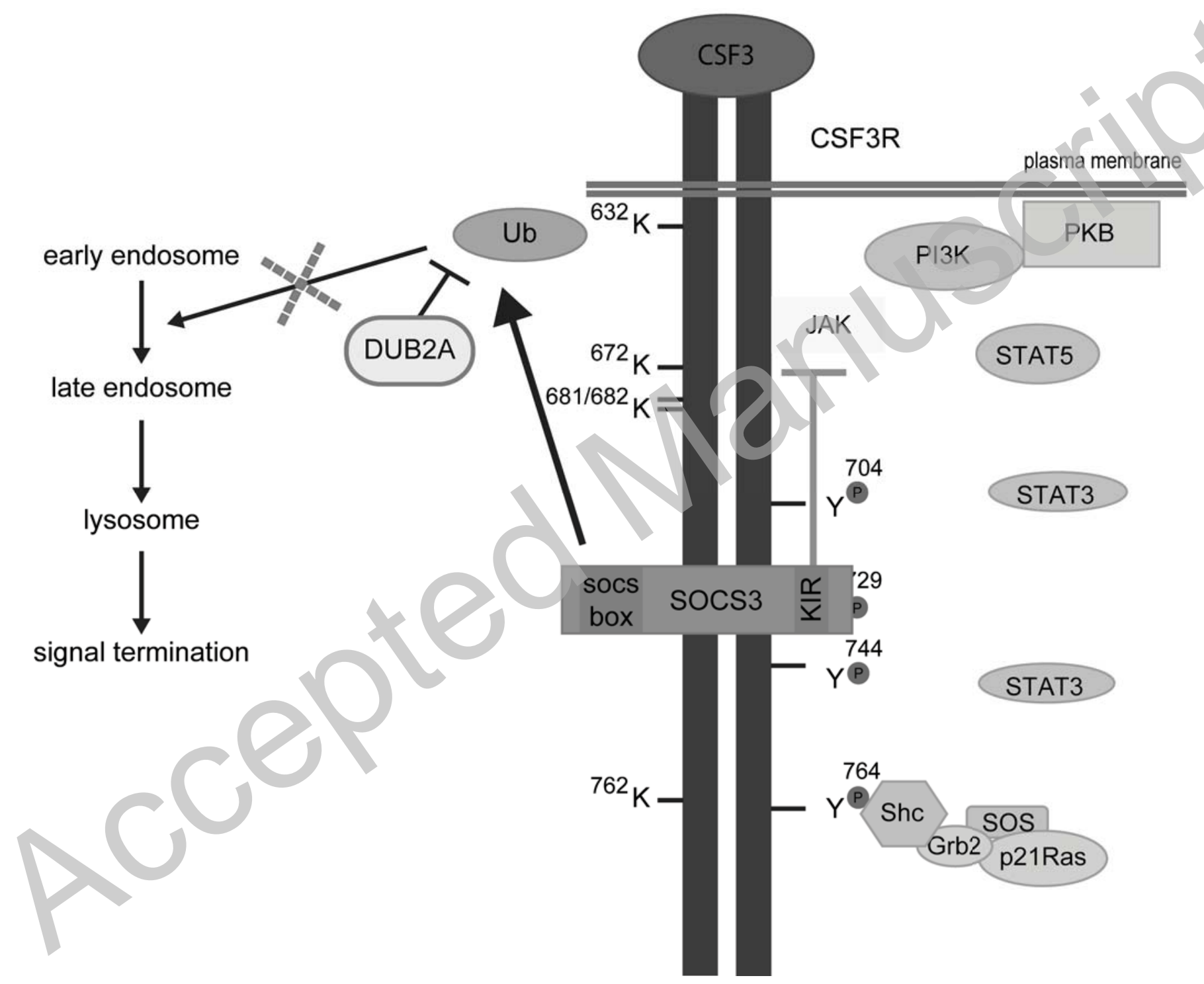

